\title{
Quantum capacitance and charge sensing of a superconducting double dot
}

\author{
N. J. Lambert, ${ }^{1, \text { a) }}$ A. A. Esmail, ${ }^{1}$ M. Edwards, ${ }^{1}$ F. A. Pollock, ${ }^{2,3}$ B. W. Lovett, ${ }^{4}$ and A. \\ J. Ferguson ${ }^{1}$ \\ 1) Microelectronics Group, Cavendish Laboratory, University of Cambridge, \\ Cambridge, СВЗ OHE, UK \\ 2) Atomic 8 Laser Physics, Clarendon Laboratory, University of Oxford, Parks Road, \\ Oxford, OX1 3PU, UK \\ 3) School of Physics \& Astronomy, Monash University, Clayton, Victoria 3800, \\ Australia \\ 4) SUPA, School of Physics and Astronomy, University of St Andrews, KY16 9SS, \\ $U K$
}

(Dated: 12 August 2016)

We study the energetics of a superconducting double dot, by measuring both the quantum capacitance of the device and the response of a nearby charge sensor. We observe different behaviour for odd and even charge states and describe this with a model based on the competition between the charging energy and the superconducting gap. We also find that, at finite temperatures, thermodynamic considerations have a significant effect on the charge stability diagram.

a) To whom correspondence should be addressed; Electronic mail: nl249@cam.ac.uk 
The ability to make small capacitance devices in which the electrostatic energy per electron is larger than the thermal energy has allowed a family of 'single electron devices' to be made and investigated, including single electron transistors (SETs), semiconductor double quantum dots, and superconducting SETs. By using capacitively coupled gate voltages to manipulate the charge occupancy, they have been exploited as, amongst other things, charge sensors $^{1-3}$, probes of chemical potential ${ }^{4}$, charge qubits $^{5,6}$ and spin qubits ${ }^{7}$. The key to controlling these devices is an understanding of the energetics of the charge states as a function of the applied gate voltages ${ }^{8}$.

A superconducting double dot (SDD) comprises two superconducting islands coupled by a Josephson junction, with each island connected to a normal metal lead by an NIS junction (Figs 2(a) and 2(b)). It is therefore similar to a semiconductor double dot, but rather than electrons, the SDD allows electrostatic manipulation of Cooper pairs and Bogoliubov quasiparticles. In this Letter, we study the charge stability diagram of an SDD. We use its quantum capacitance to probe the anticrossings between coherently coupled Cooper pair charge states, and an independent superconducting charge sensor to directly measure the charge occupancy of the device.

We start by describing the SDD theoretically, following the approaches of Tuominen ${ }^{9}$ and Lafarge ${ }^{10,11}$. Its behaviour is governed by the competition between four energy scales: the superconducting gap $(\Delta)$, the charging energies of the islands ${ }^{8}\left(E_{C R}, E_{C L}, E_{C M}\right)$, the temperature $\left(k_{B} T\right)$ and the Josephson energy of the middle tunnel junction $\left(E_{J}\right)$. We calculate the Helmholtz free energy for different charge states of the device, $F=U-T S$, where $U$ is the internal energy of the system and $S$ is the entropy. We label the charge states $(m, n)$ where $m(n)$ is the total offset charge from an arbitrarily chosen even parity state on the left (right) island. For even parity states the ground state is a Cooper pair condensate. If, however, either $m$ or $n$ is odd, then the associated quasiparticle has a momentum degree of freedom and so the quasiparticle states form a continuous band.

To find the internal energy of the device we solve the Hamiltonian for the system. Diagonal elements of the Hamiltonian are given by the sum of the electrostatic energy, $U_{E}$, which is found by treating the device as a network of capacitances ${ }^{8}$, and the energy cost of any unpaired quasiparticles. States that are related by the transfer of one Cooper pair between the islands are coupled by the matrix element $-E_{J} / 2^{12,13}$. The Hamiltonian is therefore 


$$
\begin{gathered}
\hat{H}=\sum_{m, n}\left(U_{E}+\Delta(m \bmod 2+n \bmod 2)\right)|m, n\rangle\langle m, n|- \\
\sum_{m, n \text { even }} \frac{E_{J}}{2}(|m+2, n\rangle\langle m, n+2|+| m, n+2\rangle\langle m+2, n|),
\end{gathered}
$$

and the Josephson interaction therefore leads to an anticrossing of size $E_{J}$ between states coupled by the transfer of a Cooper pair, by analogy with a Cooper pair box ${ }^{13,14}$.

We now determine the contribution of the entropy to the free energy of quasiparticle states, following the approach of Tuominen et $a l^{9}$. At low temperatures $\left(k_{B} T \ll \Delta\right)$, and in the case where a single quasiparticle is present on an island, the number of microstates available is given by $N_{\text {eff }} \approx 2 \sqrt{2 \pi} V D\left(\epsilon_{F}\right) \sqrt{\Delta k_{B} T}$, where $V$ is the island volume, $D(\epsilon)$ is the single spin density of states in the normal state and $\epsilon_{F}$ is the Fermi energy. The entropy is therefore also parity dependent, and we can now write the free energy of the system as

$$
F(m, n)=U_{E}(m, n)+\tilde{\Delta}(m \bmod 2+n \bmod 2)
$$

where $\tilde{\Delta}=\Delta-k_{B} T \ln \left(N_{\text {eff }}\right)$. The free energy of quasiparticle states decreases as temperature increases, with the energy of two-quasiparticle states decreasing at twice the rate of single-quasiparticle states.

We show the evolution of the calculated charge stability diagram with increasing $\tilde{\Delta} / E_{C}$ in Fig. 1, for an ideal device with no cross capacitance between $V_{1(2)}$ and island 2(1). We use the Ambegaokar-Baratoff relationship between $E_{J}$ and $\Delta, E_{J}=\frac{\Delta h}{8 e^{2} R_{m}}$. For $\tilde{\Delta} / E_{c}=0$ the stability diagram is that of a metallic double dot (Fig. 1(a)). Increasing $\tilde{\Delta} / E_{c}$ has two effects: the energy cost for quasiparticles increases, leading to charge states with odd parity reducing in extent (Fig. 1(b)); and an anticrossing opens up between even parity states. These are analogous to the anticrossings observed in semiconductor double dots ${ }^{15}$, and similarly result in a change in $\partial E / \partial V_{s}$, leading to a non-zero quantum capacitance close to the anticrossing ${ }^{16}$.

When $\tilde{\Delta} /\left(E_{c}-E_{J}\right)>\frac{1}{2}$ then the energy cost of the doubly odd parity $(1,1)$ state is high enough that it is never the ground state (Fig. 1(c)). Instead, the ground state at $V_{1}=0$, $V_{2}=0$ becomes the symmetric combination of the Cooper pair states, $\frac{1}{\sqrt{2}}(|2,0\rangle+|0,2\rangle)$. As $\tilde{\Delta} / E_{c}$ is increased still further (Fig. $1(\mathrm{~d})$ ), single quasiparticle states are also expelled from 


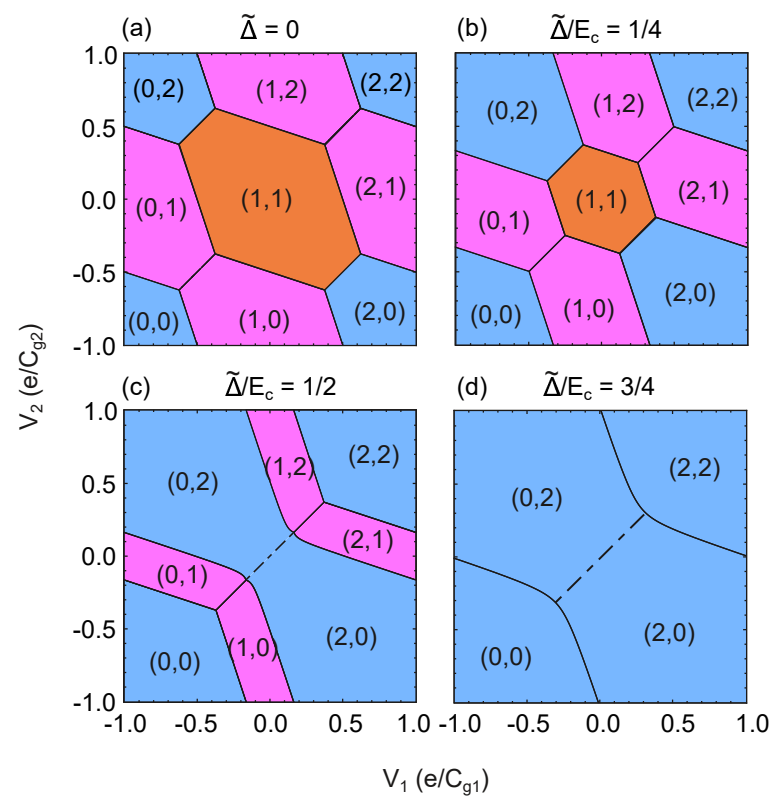

FIG. 1. Theoretical charge stability diagrams for (a) $\tilde{\Delta}=0$, (b) $\tilde{\Delta}=E_{C} / 4$, (c) $\tilde{\Delta}=E_{C} / 2$ and (d) $\tilde{\Delta}=3 E_{C} / 2$. Blue regions are Cooper pair states, purple regions have one quasiparticle present and the orange region has one quasiparticle on each island. Dotted lines in (b) and (c) correspond to anticrossings of the hybridised $(2,0)$ and $(0,2)$ levels.

the stability diagram, and it becomes $2 e$ periodic. At $V_{1}=0, V_{2}=0$, the lowest two states are the symmetric and antisymmetric Cooper pair states which form a two-level system with energy separation $E_{J}$.

We now present measurements on an SDD. Our device (Figs 2(a), 2(b)) is made using a standard three angle shadow mask process ${ }^{17}$. It consists of two Al superconducting islands separated by an $\mathrm{Al}_{2} \mathrm{O}_{3}$ tunnel junction formed by in situ oxidation of the $\mathrm{Al}$. They are connected to normal metal $\left(\mathrm{Al}_{0.98} \mathrm{Mn}_{0.02}\right)$ leads by similarly formed tunnel junctions. Close to the SDD is a $\mathrm{CPB}$, formed by a superconducting $\mathrm{Al}$ island tunnel coupled to a superconducting Al lead. The two devices are fabricated in one set of depositions, with an artefact of one of the SDD islands forming the CPB. In order to protect the CPB against quasiparticle poisoning, it is chosen to be thinner $(15 \mathrm{~nm})$ than the lead $(25 \mathrm{~nm})$, resulting in it having a larger superconducting gap ${ }^{18,19}$, and presenting a barrier to quasiparticles from the lead ${ }^{20,21}$. Electrostatic gates are also defined, to allow voltages $V_{1}, V_{2}$ and $V_{c}$ to control the electrochemical potentials of the SDD and CPB. A fourth gate is not used in these experiments. 
(a)

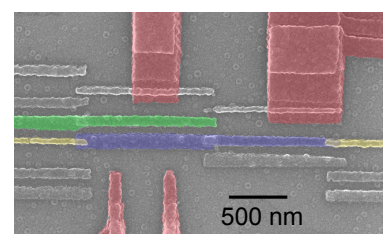

(c)

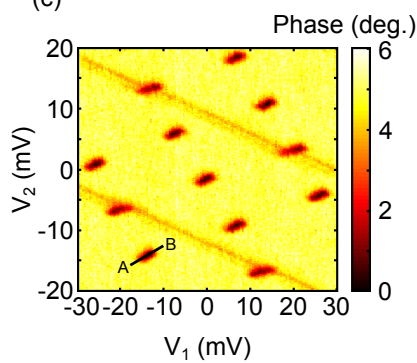

(b)

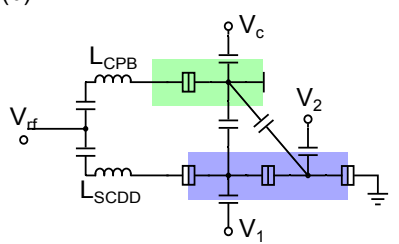

(d)

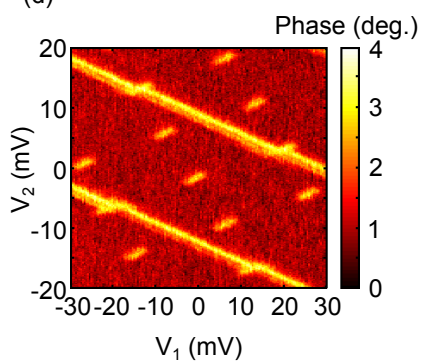

FIG. 2. The superconducting double dot and Cooper pair box. (a) False colour electron micrograph of the device. The SDD is shown in blue, the CPB in green, and red regions are the electrostatic gates. Metallic leads are yellow. Other features are artefacts of the multiple angle evaporation. (b) Schematic of the experiment. The rf signal is incident on resonant inductors $L_{\mathrm{SDD}}$ and $L_{\mathrm{CPB}}$. Potentials $V_{1}, V_{2}$ and $V_{c}$ are used to control the electrochemical potentials of the SDD and CPB. (c) Phase response at $f_{\mathrm{SDD}}$ as a function of $V_{1}$ and $V_{2}$ with $V_{c}$ grounded, showing the quantum capacitance of the SDD, and a smaller response due to the quantum capacitance of the CPB. The line $\mathrm{A} \rightarrow \mathrm{B}$ shows the crossection used in Fig. 3. (d) Phase response at $f_{\mathrm{CPB}}$ as a function of $V_{1}$ and $V_{2}$ with $V_{c}$ grounded. Here the CPB dominates the response, and the signal from the SDD is reduced.

We measure the complex impedances of the SDD and CPB mounted on the mixing chamber of a dilution fridge $(T=30 \mathrm{mK})$ using rf reflectometry. Their leads are bonded to separate lumped element inductors with resonant frequencies of $f_{\mathrm{CPB}}=298 \mathrm{MHz}$ and $f_{\mathrm{SDD}}=350 \mathrm{MHz}$ (Fig. 2(b)), allowing the two components to be probed independently. A carrier wave of power $-105 \mathrm{dBm}$ at one of the two resonant frequencies is incident upon the resonators, and the reflected power amplified at $4 \mathrm{~K}$ and room temperature before being demodulated to retrieve the phase and amplitude of the reflected signal.

We take care to minimize the nonequilibrium quasiparticle population generated by stray radiation. The sample is mounted in a light-tight copper box, coated with carbon/carbide loaded epoxy. We also surround the box with Eccosorb microwave absorbent material. Coaxial cables to the sample box are attenuated at the $1 \mathrm{~K}$ and $30 \mathrm{mK}$ stages of the dilution 
fridge. The gates are connected to twisted pairs with low-pass $\left(f_{\text {cutoff }}=10 \mathrm{kHz}\right)$ filters at $30 \mathrm{mK}$.

The charging energies of the two islands of the SDD are determined from Coulomb diamonds to be $\approx 275 \mu \mathrm{eV}$. We find the mean value of the superconducting gap for the islands to be $225 \mu \mathrm{eV}$. We expect the thinner one to have a larger gap ${ }^{19}$, but cannot discriminate between the two islands. The total resistance of the SDD is $\approx 1 \mathrm{M} \Omega$.

In Figs 2(c) and 2(d) we show the phase response for the SDD $(2(\mathrm{c}))$ and CPB $(2(\mathrm{~d}))$ as a function of $V_{1}$ and $V_{2}$, with $V_{c}$ grounded. Two sets of features are visible in each case and we ascribe both to the quantum capacitances of the two devices. The diagonal lines are due to the quantum capacitance near the charge transitions of the Cooper pair box ${ }^{13}$ and the other features are the result of the quantum capacitance of the SDD. We see the same features in both plots, because the devices are capacitatively coupled; the visibility of the feature associated with each device is enhanced when that device is probed directly.

In Figs 2(c) and 2(d) we are solely sensitive to the capacitance of the device, and hence see only the quantum capacitance due to the anticrossings between even parity charge states mediated by Cooper pair transfer between the islands. Because these anticrossings are visible, the device must be in one of the two low charging energy regimes shown in Fig. 1(c) and Fig. 1(d). However, we are not able to infer the presence or otherwise of quasiparticle states from these measurements.

For the rest of this Letter, we use $V_{c}$ to compensate for the action of $V_{1}$ and $V_{2}$ on the charge sensor; either to hold it near an operating point (in charge sensing measurements) or to hold it in a particular charge state, so that charge transitions in the CPB do not affect the SDD. We first tune the charge sensor away from any transitions, and concentrate on the response of the resonator coupled directly to the double dot.

In Fig. 3(a) and 3(b) we show measured phase along the line $A \rightarrow B$ in Fig. 2(d) as a function of applied in-plane field, $B$, and temperature. In both cases, the capacitance is suppressed, as the superconducting gap is decreased. Along with the data in 2(c) and 2(d) and the doubling of the stability diagram period under large applied fields $(B=2 \mathrm{~T})$, this suggests that, for $B=0$ and our base temperature of $30 \mathrm{mK}$, the energy scales of our device are such that $\tilde{\Delta} /\left(E_{c}-E_{J}\right)>\frac{1}{2}$, corresponding to the regime shown in either $1(\mathrm{c})$ or $1(\mathrm{~d})$.

We now use the model described above to calculate the equilibrium thermal occupancy of the different charge states along this cross-section as a function of magnetic field and temper- 

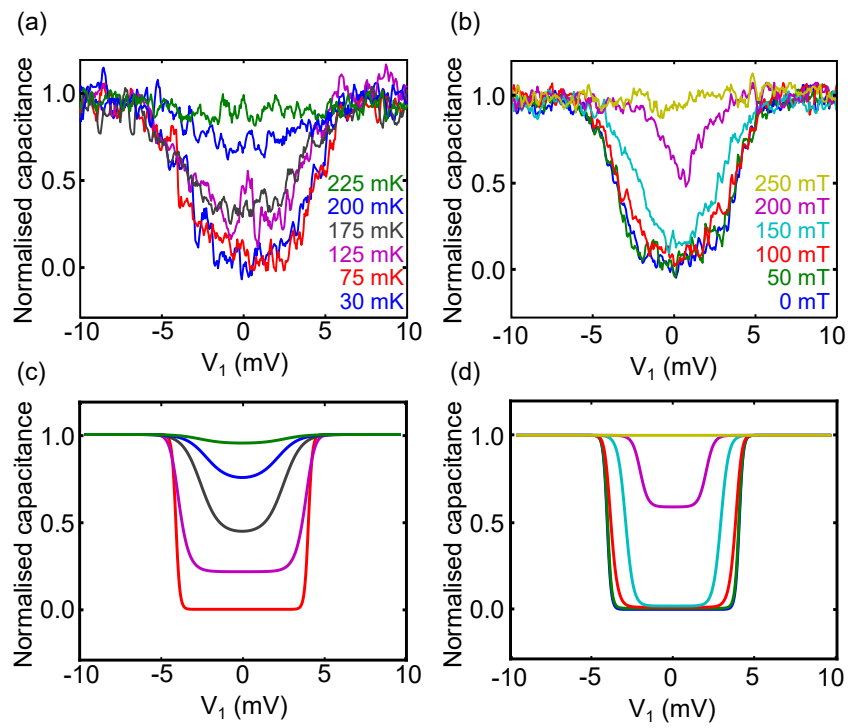

FIG. 3. Quantum capacitance. (a) Normalised device capacitance along A $\rightarrow$ B in Fig. 2(c) for increasing temperature. (b) Normalised capacitance along the same line for increasing magnetic field. In (a) and (b) $V_{c}$ is used to compensate for the action of $V_{1}$ and $V_{2}$, and holds the CPB in a single charge state. (c) and (d) Calculated normalised device capacitance weighted by state occupancy for the temperatures (c) and magnetic fields (d) in (a) and (b). In (c), we assume an base electron temperature of $75 \mathrm{mK}$, and therefore no curve is plotted for $30 \mathrm{mK}$.

ature, and hence the expected capacitance signal. We assume a base electron temperature of $75 \mathrm{mK}$. Two states contribute to the signal. The $\frac{1}{\sqrt{2}}(|2,0\rangle+|0,2\rangle)$ state has positive quantum capacitance, whilst the $\frac{1}{\sqrt{2}}(|2,0\rangle-|0,2\rangle)$ state has negative quantum capacitance of the same magnitude. This higher lying state is never significantly occupied in our experiments. The normalised expected signals are plotted in Figs 3(c) and 3(d). Increasing temperature or increasing magnetic field will suppress $\tilde{\Delta}$. However, we see a difference in the dependence of the quantum capacitance signal on these two parameters; this is due to the increased thermal occupancy of higher energy states in the case of increasing temperature. Our model is in qualitative agreement with our measurements. However, because the measurement is heavily averaged, broadening of the quantum capacitance due to $1 / f$ charge noise from a background of two level fluctuators is also present in our experimental results, and is not included in our theory.

Our quamtum capacitance data show we are in one of the two regimes shown in Fig. 1(c) and Fig. 1(d), in which the anticrossings are visible. To verify our conclusions, we now 
describe charge sensing measurements on the double dot. The charge state of a single electron device can be measured directly using a charge sensor, which has an impedance highly sensitive to the local charge distribution. For some charge sensors such as SETs ${ }^{2,3}$, the real part of the impedance is measured. Alternatively, charge sensors can rely on a change to the imaginary part of their impedance, by either a capacitance ${ }^{22}$ or inductance ${ }^{23}$ sensitive to the electrostatic environment. This approach offers a way to beat the shot noise limit for dissipative charge $\operatorname{sensors}^{24}$ and reduces back action on the system to be measured $^{25,26}$.

In Fig. 4(a) we show the phase response of $L_{\mathrm{CPB}}$ as a function of $V_{1}$ and $V_{2}$. We observe a hexagonal stability diagram, characteristic of electrostatically coupled double dots, and by comparing the gate periodicity to the normal state stability diagram measured at $B \approx 2 \mathrm{~T}$ we find that it is $2 e$ periodic. We also observe here the quantum capacitance of the SDD at the inter-island charge transitions, as in Fig. 2(d).

In Fig. 4(b) we show the phase along the line $\mathrm{A} \rightarrow \mathrm{B}$ in Fig. 4(a) for applied magnetic field values between $0 \mathrm{mT}$ and $100 \mathrm{mT}$. In this part of the stability digram, the charge sensor is tuned such that the $(0,0)$ and $(0,2)$ give similar signals in order to maximise the contrast with the $(0,1)$ state, which is observed between the $(0,0)$ and $(0,2)$ states. This confirms that we are the regime shown in Fig. $1(\mathrm{c})$ at $B=0$. As $B$ increases, $\tilde{\Delta}$ decreases, and the odd parity $(0,1)$ state increases in size, although most of the stability diagram is still even parity. At $150 \mathrm{mT}$ and higher, the periodic behaviour of the CPB is suppressed, and it can no longer act as a charge sensor.

In conclusion, we have theoretically and experimentally studied the charge stability diagram of a superconducting double dot. We find that the behaviour of the SDD depends on the competition between the charging energies and the gap, and we note that the ground state of the system can be tailored to include both single and double quasiparticle states. A device with such states could be used as an electrostatic Cooper pair splitter which, in contrast to previous work ${ }^{27-29}$, would retain the split pair. Alternatively, if the splitting is driven by incident radiation it may be usable as a tunable detector for microwave light ${ }^{16}$. 

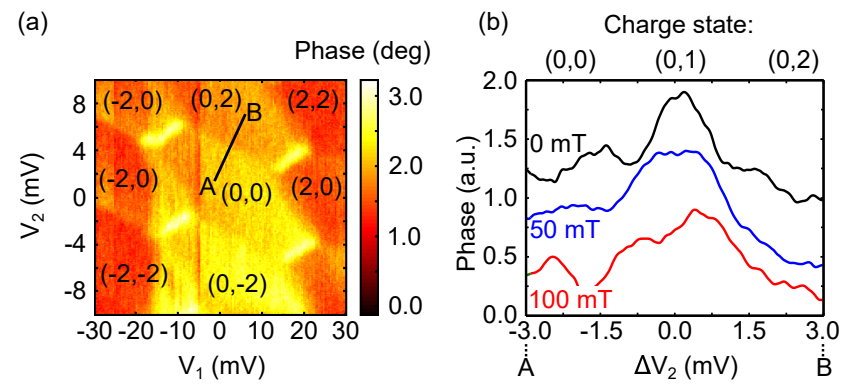

FIG. 4. Charge sensing. (a) Phase response at $f_{\mathrm{CPB}}$ with $V_{c}$ acting to hold the CPB close to a charge transition, and $B=0$. A honeycomb charge stability pattern is observed, with a background phase gradient due to the imperfect compensation of the action of $V_{1}$ and $V_{2}$ by $V_{c}$. (b) Normalised phase response at $f_{\mathrm{CPB}}$ along $\mathrm{A} \rightarrow \mathrm{B}$, for increasing magnetic fields. As magnetic field increases, the central plateau corresponding to the $(0,1)$ charge cell enlarges.

\section{ACKNOWLEDGMENTS}

We acknowledge support from Hitachi Cambridge Laboratory, and EPSRC Grant No. EP/K027018/1. A.J.F. is supported by a Hitachi Research fellowship.

\section{REFERENCES}

${ }^{1}$ G. Zimmerli, T. M. Eiles, R. L. Kautz, and J. M. Martinis, Appl. Phys. Lett. 61, 237-239 (1992).

${ }^{2}$ R. J. Schoelkopf, P. Wahlgren, A. A. Kozhevnikov, P. Delsing, and D. E. Prober, Science (80-. ). 280, 1238-1242 (1998).

${ }^{3}$ C. Barthel, M. Kjærgaard, J. Medford, M. Stopa, C. M. Marcus, M. P. Hanson, and A. C. Gossard, Phys. Rev. B 81, 161308(R) (2010).

${ }^{4}$ C. Ciccarelli, L. P. Zârbo, A. C. Irvine, R. P. Campion, B. L. Gallagher, J. Wunderlich,

T. Jungwirth, and A. J. Ferguson, Appl. Phys. Lett. 101, 122411 (2012).

${ }^{5}$ Y. Nakamura, Y. A. Pashkin, and J. S. Tsai, Nature 398, 786-788 (1999).

${ }^{6}$ T. Hayashi, T. Fujisawa, H. Cheong, Y. Jeong, and Y. Hirayama, Phys. Rev. Lett. 91, 226804 (2003).

${ }^{7}$ J. R. Petta, A. C. Johnson, J. M. Taylor, E. A. Laird, A. Yacoby, M. D. Lukin, C. M. Marcus, M. P. Hanson, and A. C. Gossard, Science (80-. ). 309, 2180-2184 (2005). 
${ }^{8}$ W. G. van der Wiel, S. De Franceschi, J. M. Elzerman, T. Fujisawa, S. Tarucha, and L. P. Kouwenhoven, Rev. Mod. Phys. 75, 1-33 (2002).

${ }^{9}$ M. T. Tuominen, J. M. Hergenrother, T. S. Tighe, and M. Tinkham, Phys. Rev. Lett. 69, 1997-2000 (1992).

${ }^{10}$ P. Lafarge, P. Joyez, D. Esteve, C. Urbina, and M. H. Devoret, Nature 365, 422-424 (1993).

${ }^{11}$ P. Lafarge, P. Joyez, D. Esteve, C. Urbina, and M. H. Devoret, Phys. Rev. Lett. 70, 994-997 (1993).

${ }^{12}$ K. K. Likharev and A. B. Zorin, J. Low Temp. Phys. 59, 347-382 (1985).

${ }^{13}$ T. Duty, G. Johansson, K. Bladh, D. Gunnarsson, C. Wilson, and P. Delsing, Phys. Rev. Lett. 95, 206807 (2005).

${ }^{14}$ M. A. Sillanpää, T. Lehtinen, A. Paila, Y. Makhlin, L. Roschier, and P. J. Hakonen, Phys. Rev. Lett. 95, 206806 (2005).

${ }^{15}$ K. D. Petersson, C. G. Smith, D. Anderson, P. Atkinson, G. A. C. Jones, and D. A. Ritchie, Nano Lett. 10, 2789-93 (2010).

${ }^{16}$ N. J. Lambert, M. Edwards, A. A. Esmail, F. A. Pollock, S. D. Barrett, B. W. Lovett, and A. J. Ferguson, Phys. Rev. B 90, 140503 (2014).

${ }^{17}$ G. J. Dolan, Appl. Phys. Lett. 31, 337 (1977).

${ }^{18}$ T. Yamamoto, Y. Nakamura, Y. A. Pashkin, O. Astafiev, and J. S. Tsai, Appl. Phys. Lett. 88, 212509 (2006).

${ }^{19}$ N. A. Court, A. J. Ferguson, and R. G. Clark, Supercond. Sci. Technol. 21, 015013 (2008).

${ }^{20}$ J. Aumentado, M. Keller, J. Martinis, and M. Devoret, Phys. Rev. Lett. 92, 066802 (2004).

${ }^{21}$ S. J. MacLeod, S. Kafanov, and J. P. Pekola, Appl. Phys. Lett. 95, 052503 (2009).

${ }^{22}$ A. C. Betz, R. Wacquez, M. Vinet, X. Jehl, A. L. Saraiva, M. Sanquer, A. J. Ferguson, and M. F. Gonzalez-Zalba, Nano Lett. 15, 4622-4627 (2015).

${ }^{23}$ M. A. Sillanpää, L. Roschier, and P. J. Hakonen, Phys. Rev. Lett. 93, 066805 (2004).

${ }^{24}$ M. A. Sillanpää, L. Roschier, and P. J. Hakonen, Appl. Phys. Lett. 87, 092502 (2005).

${ }^{25}$ B. Turek, K. Lehnert, A. Clerk, D. Gunnarsson, K. Bladh, P. Delsing, and R. Schoelkopf, Phys. Rev. B 71, 193304 (2005).

${ }^{26}$ D. Harbusch, D. Taubert, H. P. Tranitz, W. Wegscheider, and S. Ludwig, Phys. Rev. Lett. 104, 196801 (2010). 
${ }^{27}$ L. Hofstetter, S. Csonka, J. Nygård, and C. Schönenberger, Nature 461, 960-963 (2009). ${ }^{28}$ L. G. Herrmann, F. Portier, P. Roche, A. L. Yeyati, T. Kontos, and C. Strunk, Phys. Rev. Lett. 104, 026801 (2010).

${ }^{29}$ A. Das, Y. Ronen, M. Heiblum, D. Mahalu, A. V. Kretinin, and H. Shtrikman, Nat. Commun. 3, 1165 (2012). 